\title{
Mathematical Modeling of Multi-Element Antenna Arrays with Chiral Metamaterials Substrates Using Singular Integral Equations
}

\author{
Dmitriy Klyuev ${ }^{1}$, Anatoly Neshcheret ${ }^{1}$, Oleg Osipov ${ }^{1}$, and Aleksandr Potapov ${ }^{2,3, *}$ \\ ${ }^{1}$ Volga State University of Telecommunications and Informatics, RU-443010, Samara, Russia \\ ${ }^{2}$ Kotelnikov Institute of Radioengineering and Electronics, Russian Academy of Sciences, RU-125009, Moscow, Russia \\ ${ }^{3}$ E JNU-IREE Joint Laboratory of Fractal Method \& Signal Processing, Department of Electronic Engineering, College of Information \\ Science and Technology, JiNan University, Guangzhou, China
}

\begin{abstract}
In this paper, a physical model of the multi-element antenna arrays (MEAA) has been considered and a self-consistent numerical method for solving the problem of current distribution on the MEAA surface with chiral metamaterials substrate has been proposed. The algorithm of the input admittance matrix elements for chiral layer based on the conductive left and right-handed helices has been developed and elements of the matrix surface impedances for investigated structures have been found. A set of singular integral equations with a Cauchy kernel for calculating the current density on the surface of the MEAA has been derived. A numerical solution of the set is a well-posed in the sense of Hadamard. Calculations of current distribution on the MEAA surface, impedance characteristics of a three-element antenna array and the dependence of the isolation levels between emitters on the chirality parameter have been performed and analysed. It has been shown that the use of chiral substrates can substantially increase the isolation between emitters.
\end{abstract}

\section{Introduction}

One of the most promising approach to the antennas and antenna arrays (MEAA) modernization is using of chiral metamaterials in their design [1]. The analysis of the literature shows that the use of metamaterials in antenna technology can improve their electrical and massdimensional characteristics [2]. Research s of multielement antenna arrays based on such metamaterials is of great interest because they allow increasing the isolation between emitters and, as a result, improving electromagnetic compatibility (EMC) [3]. The use of such antenna arrays also allows increasing throughput in MIMO systems [4]. In addition, the recent work [5] the results of a study of reducing the effect between two emitters by using metamaterials made in the form of fractal structures [6,7] have been presented..

However, at present time, there are a number of difficulties associated with electrodynamic analysis and synthesis of such antenna arrays based on chiral metamaterials. Most studies of such structures are carried out by using electrodynamic modeling complexes, such as FEKO, CST Studio, HFSS. The use of this approach is typically associatedwith a number of difficulties . The main difficulties are the construction of correct models of the studied antennas and the choice of the optimal sampling step. In addition, the software modelling these systems is typically very demanding on computational resources.
Another approach involves the application of the moments method for the electrodynamic analysis of antenna array data based on chiral metamaterials [8, 9, 10]. The disadvantage of this approach is a need to solve Fredholm integral equations of the first kind, which belong to the class of incorrect mathematical problems in the sense of Hadamard.

The aim of the work is to develop a method of electrodynamic analysis of multi-element strip antenna arrays based on chiral metamaterials thatprovides high accuracy of calculations and requires relatively small computing power.

Based on the previous studies of single-element microstrip antennas with substrates of chiral metamaterials [11,12,13], a system of singular integral equations (SIE) was obtained for unknown functions of the distribution of current densities over emitters of a multi-element antenna array based on chiral metamaterials. The dependences of the input impedance of a three-element antenna array on the normalized arm length of the emitter were derived, as well as the dependence of the isolation between the emitters on the chirality parameter. It was shown that for some values of the chirality parameter, the level of isolation is higher.

The electrodynamic analysis method of multielement antenna arrays based on chiral metamaterials is of high practical importance, because it allows calculating their impedance, spatial and polarization 
characteristics with a high accuracy at low computational costs.

\section{Formulation of problem}

Figure 1 shows a multi-element strip antenna array located on a chiral metamaterial substrate with thickness $d$ and with macroscopic parameters $\varepsilon_{1}, \mu_{1}, \chi$. Dielectric half-space with parameters $\varepsilon_{2}, \mu_{2}$ is located above this chiral substrate. Macroscopic parameters $\varepsilon_{1}$, $\varepsilon_{2}, \mu_{1}, \mu_{2}$, are the relative permittivities and magnetic permeabilities of the media, respectively.

On the surface of the chiral substrate there is an arbitrary number of infinitely thin and perfectly conducting rectangular strips (emitters), the length of which is $2 l_{n}$, where $n$ is the serial number of the emitter. The width of these emitters is $2 a_{n}$ and the distance between them is $\Delta_{n}$. The width of the emitters gaps is $2 b_{n}$.

The emitters are excited by harmonic sources of electromotive force applied to the gaps of the emitters, due to which currents are distributed so that the resulting electromagnetic field satisfies the boundary conditions (1), Maxwell's equations, and also the radiation condition at infinity. In this case the functions of the current densities distribution are continuous both on the surfaces of the emitters and in the gaps: $\eta^{n}(x, y)$, where $x \square\left[-a_{n}, a_{n}\right], y \square\left[-l_{n}, l_{n}\right]$.

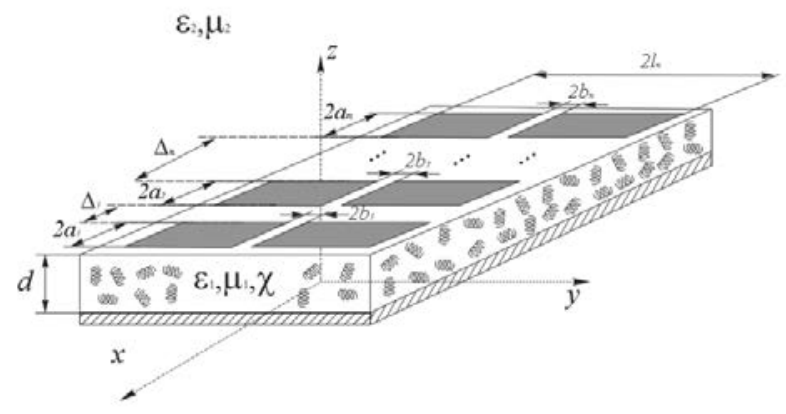

Fig. 1. Multi-element strip antenna array

We assume that the width of the emitters is much less than wavelength $\left(2 a_{n}<<\lambda\right)$, so that the transverse components of the current densities can be neglected $\left(\eta_{x}^{n}=0\right)$. It is also assumed that the tangential components of the strengths of external electric fields have one component $\overrightarrow{\mathbf{E}} \boldsymbol{\tau}_{\mathbf{n}}^{\text {ext }}=\left(0, E y_{n}^{\text {ext }}, 0\right)$.

The boundary conditions are written as follows:

$$
\begin{gathered}
\eta_{y}^{n}\left(x+\Delta_{n-1},-l_{n}\right)=\eta_{y}^{n}\left(x+\Delta_{n-1}, l_{n}\right)=0, \\
\overrightarrow{\mathbf{E}} \boldsymbol{\tau}_{\mathbf{n}}\left(x+\Delta_{n-1}, y\right)=0, \text { provided } x+\Delta_{n-1} \in\left[-a_{n}, a_{n}\right], \\
y \in\left[-l_{n},-b_{n}\right] \cup\left[b_{n}, l_{n}\right](1) \\
\overrightarrow{\mathbf{E}} \boldsymbol{\tau}_{\mathbf{n}}\left(x+\Delta_{n-1}, y\right)=-\overrightarrow{\mathbf{E}} \boldsymbol{\tau}_{\mathbf{n}}^{\mathrm{ext}}, \text { provided }
\end{gathered}
$$

$$
x+\Delta_{n-1} \in\left[-a_{n}, a_{n}\right], y \in\left[-b_{n}, b_{n}\right] .
$$

\section{Surface impedance matrix}

The Fourier transform $\overrightarrow{\mathbf{T}}_{\tau}=\left\{T_{x}, T_{y}\right\}$ of the tangential component of the electric field $\overrightarrow{\mathbf{E}} \boldsymbol{\tau}$ and the Fourier transform of the current density $\overrightarrow{\mathbf{F}}=\left\{F_{x}, F_{y}\right\}$ on the emitters $\overrightarrow{\boldsymbol{\eta}}^{\mathbf{n}}$ are also connected through the matrix of surface impedances $[\mathbf{z}]$ on the surfaces of the emitters (in plane $z=d$ ):

$$
\left[\begin{array}{l}
T_{y} \\
T_{x}
\end{array}\right]=\left[\begin{array}{ll}
Z_{11} & Z_{12} \\
Z_{21} & Z_{22}
\end{array}\right]\left[\begin{array}{l}
F_{y} \\
F_{x}
\end{array}\right],
$$

where $Z_{i j} \quad(i, j=1,2)$ are the elements of the surface impedance, which are functions of the Fourier space variables $\beta, h: Z_{i j}=Z_{i j}(\beta, h)$.

Since it is the Fourier transforms of the field strength and surface current density that are used, the vectors of the electric field $\overrightarrow{\mathbf{E}}$, magnetic field $\overrightarrow{\mathbf{H}}$ and surface current density $\overrightarrow{\boldsymbol{\eta}}$ were decomposed into Fourier integrals by coordinates $x$ and $y$ :

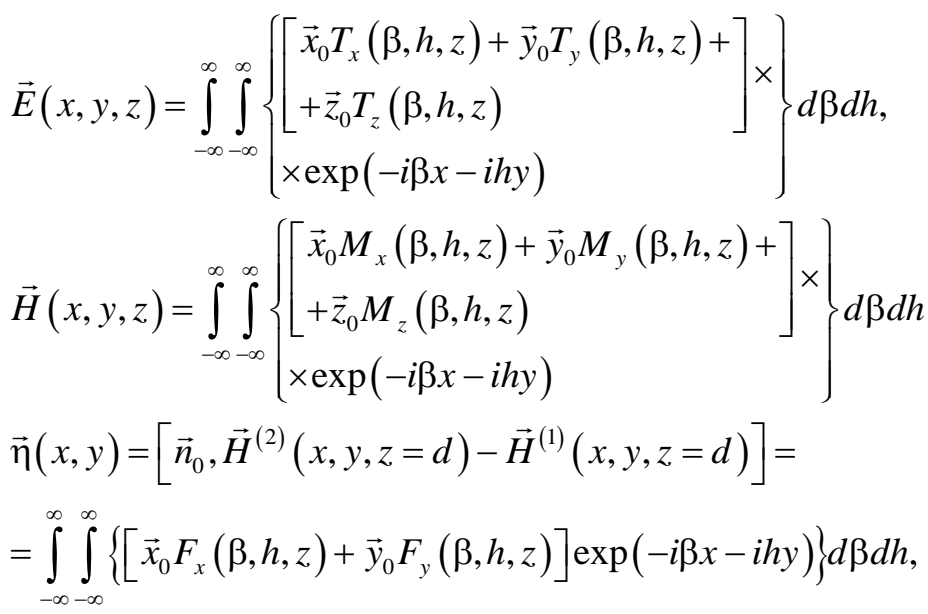

where

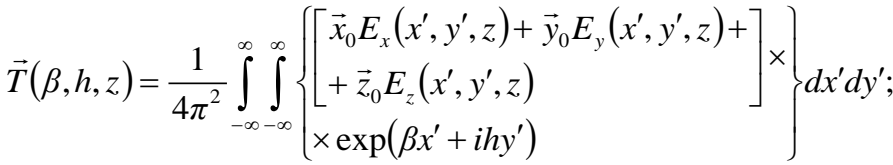

$$
\begin{aligned}
& \vec{M}(z, \beta, h)=\frac{1}{4 \pi^{2}} \int_{-\infty}^{\infty} \int_{-\infty}^{\infty}\left\{\begin{array}{l}
{\left[\begin{array}{l}
\vec{x}_{0} H_{x}\left(x^{\prime}, y^{\prime}, z\right)+\vec{y}_{0} H_{y}\left(x^{\prime}, y^{\prime}, z\right)+ \\
+\vec{z}_{0} H_{z}\left(x^{\prime}, y^{\prime}, z\right) \\
\times \exp \left(\beta x^{\prime}+i h y^{\prime}\right)
\end{array}\right] \times}
\end{array}\right\} d x^{\prime} d y^{\prime} ;
\end{aligned}
$$

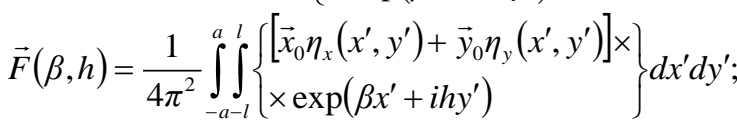

In (3) $\vec{x}_{0}, \vec{y}_{0}, \vec{z}_{0}$ are the unit vectors of the Cartesian coordinate system; $\left\{E_{x}, E_{y}, E_{z}\right\}$, 
$\left\{H_{x}, H_{y}, H_{z}\right\}, \quad\left\{\eta_{x}, \eta_{y}\right\}$ are components of the vectors of the electric field $\overrightarrow{\mathbf{E}}$, magnetic field $\overrightarrow{\mathbf{H}}$ and surface current density $\overrightarrow{\boldsymbol{\eta}}$, respectively; $\left\{T_{x}, T_{y}, T_{z}\right\}$, $\left\{M_{x}, M_{y}, M_{z}\right\},\left\{F_{x}, F_{y}\right\}$ are components of Fourierimage vectors of the electric field $\overrightarrow{\mathbf{E}}$, magnetic field $\overrightarrow{\mathbf{H}}$ and surface current density $\overrightarrow{\boldsymbol{\eta}}$, respectively. In (4), considering that the surface density of electric current $\overrightarrow{\boldsymbol{\eta}}$ is different from zero only on the surface of the MEAA: $x \in[-a, a], y \in[-l, l]$. In (4): $\vec{n}_{0}$ is the unit normal vector to the surface between the first and second region; $\vec{H}^{(1)}, \vec{H}^{(2)}$ are the magnetic field vectors of the in region $1(z<d)$ and region $2(z>d)$, respectively.

To determine the surface impedance matrix $[Z]$, it is simpler to find the matrix surface admittances $[Y]$ :

$$
\left[\begin{array}{l}
F_{y} \\
F_{x}
\end{array}\right]=\left[\begin{array}{ll}
Y_{11} & Y_{12} \\
Y_{21} & Y_{22}
\end{array}\right]\left[\begin{array}{l}
T_{y} \\
T_{x}
\end{array}\right] \text {, }
$$

where $Y_{i j}(i, j=1,2)$ are the elements of the matrix surface admittances $[Y]$, depending on variables $\beta, h$.

Since the matrix of relations (2) and (5) it follows that the matrix $[Y]$ has an inverse matrix $[Z]$ and the relationships between the elements of these matrices are:

$$
\begin{aligned}
& Z_{11}(\beta, h)=Y_{22}(\beta, h) / \Delta(\beta, h), \\
& Z_{12}(\beta, h)=-Y_{12}(\beta, h) / \Delta(\beta, h), \\
& Z_{21}(\beta, h)=-Y_{21}(\beta, h) / \Delta(\beta, h), \\
& Z_{22}(\beta, h)=Y_{11}(\beta, h) / \Delta(\beta, h),
\end{aligned}
$$

where

$$
\Delta(\beta, h)=Y_{11}(\beta, h) Y_{22}(\beta, h)-Y_{12}(\beta, h) Y_{21}(\beta, h) .
$$

The surface admittances matrix elements of the plane $Z=d$ are determined through the input admittances matrix of a region $(z>d)$ (dielectric layer) $\left[Y^{(2)}\right]$ and the matrix of a region $(z<d)$ (chiral layer) $\left[Y^{(1)}\right]$ :

$$
Y_{i j}=Y_{i j}^{(2)}-Y_{i j}^{(1)}
$$

The input admittances matrices are introduced as the following expressions:

$$
\left[\begin{array}{l}
M_{X}^{(1,2)} \\
M_{y}^{(1,2)}
\end{array}\right]=\left[\begin{array}{cc}
Y_{11}^{(1,2)} & Y_{12}^{(1,2)} \\
Y_{21}^{(1,2)} & Y_{22}^{(1,2)}
\end{array}\right]\left[\begin{array}{c}
T_{y}^{(1,2)} \\
T_{X}^{(1,2)}
\end{array}\right],
$$

where $T_{x}^{(1)}, T_{y}^{(1)}, M_{x}^{(1)}, M_{y}^{(1)}$ are the Fourier transforms of the electric and magnetic fields tangential components in the plane $z=d$ of region $(z<d)$ and $T_{x}^{(2)}, T_{y}^{(2)}, M_{x}^{(2)}, M_{y}^{(2)}$ are the Fourier transforms of the electric and magnetic fields tangential components of the in the plane $z=d$ of region $(z>d)$.

First, we must define the input admittances elements of region $(z<d)$ (chiral layer). We write the Maxwell equations for the complex amplitudes in the Cartesian coordinate system:

$$
\begin{aligned}
& \frac{\partial E_{z}^{(1)}}{\partial y}-\frac{\partial E_{y}^{(1)}}{\partial z}=-i \omega \mu_{0} \mu_{1} H_{x}^{(1)} \pm k \chi E_{x}^{(1)}, \\
& \frac{\partial H_{z}^{(1)}}{\partial y}-\frac{\partial H_{y}^{(1)}}{\partial z}=i \omega \varepsilon_{0} \varepsilon_{1} E_{x}^{(1)} \pm k \chi H_{x}^{(1)}, \\
& \frac{\partial E_{x}^{(1)}}{\partial z}-\frac{\partial E_{z}^{(1)}}{\partial x}=-i \omega \mu_{0} \mu_{1} H_{y}^{(1)} \pm k \chi E_{y}^{(1)}, \\
& \frac{\partial H_{x}^{(1)}}{\partial z}-\frac{\partial H_{z}^{(1)}}{\partial x}=i \omega \varepsilon_{0} \varepsilon_{1} E_{y}^{(1)} \pm k \chi H_{y}^{(1)}, \\
& \frac{\partial E_{y}^{(1)}}{\partial x}-\frac{\partial E_{x}^{(1)}}{\partial y}=-i \omega \mu_{0} \mu_{1} H_{z}^{(1)} \pm k \chi E_{z}^{(1)}, \\
& \frac{\partial H_{y}^{(1)}}{\partial x}-\frac{\partial H_{X}^{(1)}}{\partial y}=i \omega \varepsilon_{0} \varepsilon_{1} E_{z}^{(1)} \pm k \chi H_{z}^{(1)},
\end{aligned}
$$

where $\varepsilon_{0}$ is the electric constant of vacuum, $\mu_{0}$ is the magnetic constant of vacuum, $\varepsilon_{1}, \mu_{1}$ are the chiral layer relative permittivity and permeability, $\chi$ is the relative chirality parameter, $k=\omega \sqrt{\varepsilon_{0} \mu_{0}}$ is the wave number for a plane wave in vacuum, $\omega$ is the frequency. Equations (9) are only valid for a chiral media, consisting of a right-handed (upper arithmetic signs) and left-handed (bottom arithmetic signs) helices. This is determined by the sign in the material equations for chiral media according to the Lindell-Sihvola formalism $[14,15]$.

We have the following equations system for the electromagnetic field in the chiral substrate:

$$
\begin{aligned}
& \nabla^{2} \vec{E}+k^{2}\left(\varepsilon_{1} \mu_{1}+\chi^{2}\right) \vec{E} \mp 2 i \omega \mu_{0} \mu_{1} k \chi \vec{H}=0, \\
& \nabla^{2} \vec{H}+k^{2}\left(\varepsilon_{1} \mu_{1}+\chi^{2}\right) \vec{H} \pm 2 i \omega \varepsilon_{0} \varepsilon_{1} k \chi \vec{E}=0,
\end{aligned}
$$

Substituting (3) into (10) and taking the derivatives of $x$ and $y$, we obtain the following system of equations for the Fourier-images $\left\{T_{x}, T_{y}, T_{z}\right\},\left\{M_{x}, M_{y}, M_{z}\right\}$ :

$$
\begin{aligned}
& \frac{\partial^{2} \vec{T}}{\partial z^{2}}+\left[k^{2}\left(\varepsilon_{1} \mu_{1}+\chi^{2}\right)-\beta^{2}-h^{2}\right] \vec{T} \mp 2 i \omega \mu_{0} \mu_{1} k \chi \vec{M}=0, \\
& \frac{\partial^{2} \vec{M}}{\partial z^{2}}+\left[k^{2}\left(\varepsilon_{1} \mu_{1}+\chi^{2}\right)-\beta^{2}-h^{2}\right] \vec{M} \pm 2 i \omega \varepsilon_{0} \varepsilon_{1} k \chi \vec{T}=0,
\end{aligned}
$$


We define $T_{y}^{(1)}$ and $M_{y}^{(1)}$ from equation (11) solutions with the boundary condition $T_{y}^{(1)}(z=0)=0$ :

$$
\begin{aligned}
& T_{y}^{(1)}= \pm C\left(\sin \left(\gamma_{R} z\right)+\sin \left(\gamma_{L} z\right)\right), \\
& M_{y}^{(1)}=C i \sqrt{\frac{\varepsilon_{0} \varepsilon_{1}}{\mu_{0} \mu_{1}}}\left(\sin \left(\gamma_{R} z\right)-\sin \left(\gamma_{L} z\right)\right),
\end{aligned}
$$

where

$$
\gamma_{R}=\sqrt{k^{2}(n+\chi)^{2}-\beta^{2}-h^{2}}, \gamma_{L}=\sqrt{k^{2}(n-\chi)^{2}-\beta^{2}-h^{2}}, n=\sqrt{\varepsilon_{1} \mu_{1}},
$$

\section{$C$ is a constant.}

We substitute expression (3) to (9) and express the components $T_{x}^{(1)}, M_{x}^{(1)}$ through $T_{y}^{(1)}, M_{y}^{(1)}$. Using these expressions and the relations (12), we obtain the values of the matrix input admittances elements on the plane $z=d$ of region $(z<d)$ (chiral layer):

$$
\begin{gathered}
Y_{11}^{(1)}(\beta, h)=-\frac{a(h)\left[i \omega \varepsilon_{0} \varepsilon_{1} v^{+}(\beta, h)-Y_{12}^{(1)}(\beta, h) w^{+}(\beta, h)\right]}{\sin \left(\gamma_{R} z\right)+\sin \left(\gamma_{L} z\right)} \\
Y_{12}^{(1)}(\beta, h)=-\frac{w^{-}(\beta, h)}{i \omega \mu_{0} \mu_{1} v^{-}(\beta, h)} \\
Y_{21}^{(1)}(\beta, h)=\frac{a(h) Y_{22}^{(1)}(\beta, h)}{\sin \left(\gamma_{R} z\right)+\sin \left(\gamma_{L} z\right)} w^{+}(\beta, h) \\
Y_{22}^{(1)}(\beta, h)=\frac{\sin \left(\gamma_{R} z\right)-\sin \left(\gamma_{L} z\right)}{i \omega \mu_{0} \mu_{1} v^{-}(\beta, h) a(h)}
\end{gathered}
$$

where

$$
\begin{aligned}
& a(h)=\frac{1}{\left(k^{2} \varepsilon_{1} \mu_{1}+k^{2} \chi^{2}-h^{2}\right)}\left(1-\frac{4 k^{4} \chi^{2} \varepsilon_{1} \mu_{1}}{\left(k^{2} \varepsilon_{1} \mu_{1}+k^{2} \chi^{2}-h^{2}\right)^{2}}\right)^{-1} ; \\
& \zeta(h)=\frac{2 k \chi}{\left(k^{2} \varepsilon_{1} \mu_{1}+k^{2} \chi^{2}-h^{2}\right)} ; \\
& s(h)=1-\frac{2 k^{2} \chi^{2}}{\left(k^{2} \varepsilon_{1} \mu_{1}+k^{2} \chi^{2}-h^{2}\right)} ; \\
& q(h)=k \chi-\frac{2 k^{3} \chi \varepsilon_{1} \mu_{1}}{\left(k^{2} \varepsilon_{1} \mu_{1}+k^{2} \chi^{2}-h^{2}\right)} ; \\
& v^{+}(\beta, h)=-s(h)\left( \pm\left(\gamma_{R} \cos \left(\gamma_{R} z\right)+\gamma_{L} \cos \left(\gamma_{L} z\right)\right)\right)+ \\
& +\zeta(h) \beta h\left(\sin \left(\gamma_{R} z\right)+\sin \left(\gamma_{L} z\right)\right) \\
& v^{-}(\beta, h)=-s(h)\left(\gamma_{R} \cos \left(\gamma_{R} z\right)-\gamma_{L} \cos \left(\gamma_{L} z\right)\right) \pm \\
& \pm \zeta(h) \beta h\left(\sin \left(\gamma_{R} z\right)-\sin \left(\gamma_{L} z\right)\right) \\
& w^{+}(\beta, h)=-\beta h\left( \pm\left(\sin \left(\gamma_{R} z\right)+\sin \left(\gamma_{L} z\right)\right)\right) \mp \\
& \mp q(h)\left( \pm\left(\gamma_{R} \cos \left(\gamma_{R} z\right)+\gamma_{L} \cos \left(\gamma_{L} z\right)\right)\right)
\end{aligned}
$$

$$
\begin{aligned}
& w^{-}(\beta, h)=\beta h\left(\sin \left(\gamma_{R} z\right)-\sin \left(\gamma_{L} z\right)\right) \pm \\
& \pm q(h)\left(\gamma_{R} \cos \left(\gamma_{R} z\right)-\gamma_{L} \cos \left(\gamma_{L} z\right)\right)
\end{aligned} .
$$

The method for determining the elements of the matrix of input admittances of the plane $z=d$ of the region $(z>d)$ (dielectric layer) is similar to this method. In the derivation of these expressions, the chirality parameter was assumed to be zero. Here we present only the final expressions:

$$
\begin{aligned}
& Y_{11}^{(2)}=-\frac{k^{2} \varepsilon_{2} \mu_{2}-\beta^{2}}{\omega \mu_{0} \mu_{2} r_{2}} ; \quad Y_{12}^{(2)}=-\frac{h \beta}{\omega \mu_{0} \mu_{2} r_{2}} ; \\
& Y_{21}^{(2)}=\frac{h \beta}{\omega \mu_{0} \mu_{2} r_{2}} ; \quad Y_{22}^{(2)}=\frac{k^{2} \varepsilon_{2} \mu_{2}-h^{2}}{\omega \mu_{0} \mu_{2} r_{2}} ;
\end{aligned}
$$

where $r_{2}=\sqrt{k^{2} \varepsilon_{2} \mu_{2}-\beta^{2}-h^{2}}, \quad \varepsilon_{2}, \quad \mu_{2}$ are the relative permittivity and permeability of dielectric layer.

By defining elements of the input admittances matrices, it is easy to obtain elements of the matrix surface admittances and impedances of the surface plane $z=d$. Using the matrix equations (2), (5), (8) and performing inverse Fourier transform, we can get expressions relating the electric and magnetic radiation field with a current density at the surface. By substituting these expressions them into the appropriate boundary these conditions are transformed into integral equations for the current density at the MEAA surface.

\section{System of singular integral equations}

Taking into account the expression for the surface impedances matrix (2) we obtain the expression for the integral representation of the field of a MEAA on the surface of the emitters:

$$
\begin{aligned}
& E_{p}^{y}(x, y)= \\
& =\sum_{n=1}^{N} \int_{-l_{n}}^{l_{n}} \int_{n-1}^{L_{n-1}-a_{n}} \eta_{n}^{y}\left(x^{\prime}, y^{\prime}\right) Z_{n p}^{\Sigma}\left(x^{\prime}, y^{\prime}, x, y\right) d x^{\prime} d y^{\prime}, \\
& Z_{n p}^{\Sigma}\left(x^{\prime}=\Delta_{n-1}, y^{\prime}, x=\Delta_{p-1}, y\right)= \\
& =\frac{1}{4 \pi^{2}} \int_{-\infty-\infty}^{\infty} \int_{11}^{\infty} Z_{11}(\beta, h) e^{-i \beta\left(x-x^{\prime}\right)} e^{-i h\left(y-y^{\prime}\right)} d \beta d h,
\end{aligned}
$$

where $p$ is a number of the emitter.

For convenience, we assume that the $x$-coordinate of the location of the first emitter coincides with the origin, i.e. equal to zero $\left(x_{1}=\Delta_{0}=0\right)$. Also the abscissa of coordinates of other emitters location will coincide with the distances between them $\left(x_{n}=\Delta_{n-1}\right)$.

Due to the emitters are rather narrow $(a<<\lambda)$ the transverse variation of the longitudinal distribution of the surface current density $\eta_{y}^{n}(x)$ can be described by the quasistatic distribution $1 / \sqrt{1-(x / a)^{2}} \quad[11,12]$ and the 
distribution functions of the current densities $\eta_{y}^{n}\left(x^{\prime}, y^{\prime}\right)$ will be described as:

$$
\eta_{n}^{y}\left(x^{\prime}, y^{\prime}\right)=\frac{f_{n}\left(y^{\prime}\right)}{\sqrt{1-\left(\left(x^{\prime}-\Delta_{n-1}\right) / a_{n}\right)^{2}}},
$$

where $f_{n}\left(y^{\prime}\right)$ are unknown functions characterizing the longitudinal distribution of surface current densities at the respective emitters.

After substituting (17) into (16) we obtain the following expression and after applying the integration procedure in parts $y^{\prime}$ to the resulting expression taking into account boundary condition $f_{n}\left(-l_{n}\right)=f_{n}\left(l_{n}\right)=0$, a transition to new functions was performed: $f_{n}^{\prime}\left(y^{\prime}\right)=d f_{n}\left(y^{\prime}\right) / d y^{\prime}:$

$$
\begin{aligned}
& E_{p}^{y}\left(\Delta_{p-1}, y\right)=-\frac{1}{4 \pi} \sum_{n=1}^{N} \int_{-I_{n}}^{l_{n}} a_{n} f_{n}^{\prime}\left(y^{\prime}\right) \times \\
& \times \int_{-\infty-\infty}^{\infty} \int_{0}^{\infty} J_{0}\left(\beta a_{n}\right) \frac{Z_{11}(\beta, h)}{i h} e^{i \beta\left(\Delta_{n-1}-\Delta_{p-1}\right)} e^{-i h\left(y-y^{\prime}\right)} d \beta d h d y^{\prime} .
\end{aligned}
$$

Expression (18) shows that improper integrals over $h$ are also divergent. To eliminate the divergence an asymptotic residue was performed, i.e. in the integrands, the addition and subtraction of the asymptotic factor $Z_{11}^{\infty}(h)$ was performed. The expression of the asymptotic factor is:

$$
Z_{11}(\beta, h) \underset{|h| \rightarrow \infty}{\longrightarrow} Z_{11}^{\infty}(h)=C_{\varepsilon, \mu, \chi}|h|,
$$

where

$$
C_{\varepsilon, \mu, \chi}=\frac{i \omega \mu_{0}\left(\mu_{1}+\mu_{2}\right)}{\left(k^{2}\left(\varepsilon_{1}+\varepsilon_{2}\right)\left(\mu_{1}+\mu_{2}\right)-(k \chi)^{2}\right)} .
$$

After a series of mathematical transformations system of singular integral representations (SIR) of the electric field was obtained, which when the boundary conditions are substituted (1), goes over the system of singular integral equations (SIE) with Cauchy-type features with respect to unknown functions characterizing the longitudinal current distributions over AR emitters based on chiral metamaterials:

$$
\begin{aligned}
& -E y_{p}^{e x t}\left(\Delta_{p-1}, y\right)=\sum_{n=1}^{N}\left\{-\frac{1}{4 \pi} \int_{-l_{n}}^{l_{n}} a_{n} f_{n}^{\prime}\left(y^{\prime}\right) \times\right. \\
& \times\left[\int_{-\infty-\infty}^{\infty} \int_{-\infty}^{\infty} J_{0}\left(\beta a_{n}\right) \frac{Z_{11}(\beta, h)-Z_{11}^{\infty}(h)}{i h} \times\right. \\
& \left.\times e^{i \beta\left(\Delta_{n-1}-\Delta_{p-1}\right)} e^{-i h\left(y-y^{\prime}\right)} d \beta d h d y^{\prime}\right]+ \\
& \left.+\frac{C_{\varepsilon, \mu, \chi}}{\pi} \zeta\left(\Delta_{p-1}-\Delta_{n-1}\right) \int_{-l_{n}}^{l_{n}} \frac{a_{n} f_{n}^{\prime}\left(y^{\prime}\right)}{y^{\prime}-y} d y^{\prime}\right\} .
\end{aligned}
$$

The normalized system of singular integral equations has the following form:

$$
\begin{aligned}
& -\sigma_{p} v_{p}(t)=\sum_{n=1}^{N}\left[\int_{-I_{n}}^{l_{n}} f_{n}^{\prime}\left(t^{\prime}\right) K_{n p}\left(t, t^{\prime}\right) d t^{\prime}+\right. \\
& \left.+a_{n} \zeta\left(\Delta_{p-1}-\Delta_{n-1}\right) \frac{1}{\pi} \int_{-I_{n}}^{I_{n}} \frac{f_{n}^{\prime}\left(t^{\prime}\right)}{t^{\prime}-t} d t^{\prime}\right] .
\end{aligned}
$$

where

$$
\begin{aligned}
& K_{n p}\left(t, t^{\prime}\right)=-\frac{l_{p} / a_{n}}{2 \pi} \int_{-\infty}^{\infty} \frac{1}{\xi} \Delta g_{n p}(\xi) e^{-i \frac{l_{p}}{a_{n}} \xi\left(t-t^{\prime}\right)} d \xi ; \\
& \Delta g_{n p}(\xi)=\frac{1}{i} \frac{1}{C_{\varepsilon, \mu, \chi}^{n}} \times \\
& \times \int_{0}^{\infty} J_{0}(\alpha)\left[-\left(\frac{2 \pi a_{n}}{\lambda}\right)^{2} Z_{11}^{n}(\alpha, \xi)-C_{\varepsilon, \mu, \chi}^{n}|\xi|\right] e^{i \frac{\left(\Delta_{n-1}-\Delta_{p-1}\right)}{a_{n}} d} d \alpha ; \\
& C_{\varepsilon, \mu, \chi}^{n}=-\frac{\mu_{1}+\mu_{2}}{\left(\varepsilon_{1}+\varepsilon_{2}\right)\left(\mu_{1}+\mu_{2}\right)-\chi^{2}} ; \\
& E y_{p}^{e x t}\left(\Delta_{p-1}, t\right)=E 0_{p} v_{p}(t) ; E 0_{p}=\frac{V_{p}}{2 b_{p}} ; \\
& \sigma_{p}=i \pi \frac{l_{p}}{\lambda} \frac{1}{Z_{c}} \frac{l_{p}}{b_{p}} \frac{1}{C_{\varepsilon, \mu, \chi}^{n}} V_{p} ;
\end{aligned}
$$

$v_{n}(t)$ are voltage profiles in the gaps of the emitters;

$Z_{c}$ is characteristic resistance.

\section{Solution of a system of singular integral equations}

The obtained system of singular integral equations was solved using by moments method, which with a sufficient number of members of the approximating series allows one to obtain correct results of calculating the characteristics of the MEAA with sufficiently high accuracy $[11,12,13]$.

Unknown functions characterizing the longitudinal distributions of surface currents on emitters were presented in the form of a series consisting of Chebyshev polynomials of the first kind:

$$
f_{n}^{\prime}\left(t^{\prime}\right)=\sum_{k=1}^{\infty} \frac{A_{k}^{n} T_{k}\left(t^{\prime}\right)}{\sqrt{1-t^{\prime 2}}}
$$

In order to simplify the procedure of numerical integration of the exponential in the kernel of expression (21) we decomposed them into Bessel functions and the 1st and 2nd kind Chebyshev polynomials:

$$
\begin{aligned}
& e^{-i \frac{l_{p}}{a_{n} \xi t}}=\frac{2 i}{\left(\xi l_{p} / a_{n}\right)} \sum_{v=1}^{\infty} i^{-v} k J_{v}\left(\xi l_{p} / a_{n}\right) U_{v-1}(t) \\
& e^{i \frac{l_{p}}{a_{n} t^{\prime}}}=2 \sum_{\varsigma=0}^{\infty} \frac{i^{\varsigma}}{\left(1+\delta_{0, \varsigma}\right)} J_{\varsigma}\left(\xi l_{p} / a_{n}\right) T_{\varsigma}\left(t^{\prime}\right)
\end{aligned}
$$


where $\delta_{0, \varsigma}=\left\{\begin{array}{l}1, \varsigma=0 \\ 0, \varsigma \neq 0\end{array}\right.$ is a Kronecker symbol.

After substituting expressions (22) and (23) into the system of SIE (21) and calculating in an analytical form a series of table integrals we obtain the following system of linear algebraic equations (SLAE):

$$
B_{m}^{p}=\sum_{n=1}^{N} \sum_{k=1}^{\infty} A_{k}^{n} C_{k m}^{p n},
$$

where

$$
\begin{aligned}
& B_{m}^{p}=\frac{2}{\pi} \sigma_{p} \int_{-b_{p} / l_{p}}^{b_{p} / l_{p}} \sqrt{1-t^{2}} U_{m-1}(t) N_{p}(t) d t, \\
& C_{k m}^{p n}=m i^{k-m+1} \zeta_{n, p, k, m}+a_{n} \zeta\left(\Delta_{p-1}-\Delta_{n-1}\right) \times\left\{\begin{array}{ll}
1, & m=k \\
0, & m \neq k
\end{array}\right], \\
& \zeta_{n, p, v, \varsigma}=\int_{-\infty}^{\infty} \Delta g_{n p}(\xi) \frac{J_{\zeta}\left(\xi l_{p} / a_{n}\right) J_{v}\left(\xi l_{p} / a_{n}\right)}{\xi^{2}} d \xi, \\
& \zeta\left(\Delta_{p-1}-\Delta_{n-1}\right)=\left\{\begin{array}{l}
\frac{1}{\sqrt{a_{n}^{2}-\left(\Delta_{p-1}-\Delta_{n-1}\right)^{2}}}, \quad\left|\Delta_{p-1}-\Delta_{n-1}\right|<a_{n} \\
0, \quad\left|\Delta_{p-1}-\Delta_{n-1}\right|>a_{n}
\end{array}\right.
\end{aligned}
$$

The matrix of SLAE relative to unknown coefficients $A_{k}^{n}$ has the following form:

$$
\left[A_{k}^{n}\right]=\left[B_{m}^{p}\right]\left[C_{k m}^{p n}\right]^{-1}
$$

Unknown functions characterizing the longitudinal distribution of the surface current density on the emitters are determined in accordance with the following expression:

$$
\begin{aligned}
& f_{n}(t)=\int_{-1}^{t} f_{n}^{\prime}\left(t^{\prime}\right) d t^{\prime}= \\
& =\sum_{k=1}^{\infty} \int_{-1}^{t} \frac{A_{k}^{n} T_{k}\left(t^{\prime}\right)}{\sqrt{1-t^{\prime 2}}} d t=-\sqrt{1-t^{2}} \sum_{k=1}^{\infty} \frac{A_{k}^{n}}{k} U_{k-1}(t)
\end{aligned}
$$

The current function is defined as follows:

$$
I_{y}^{n}(t)=\int_{-a}^{a} \eta_{y}^{n}(x, t) d x=\int_{-a_{n}}^{a_{n}} \frac{f_{n}(t)}{\sqrt{1-\left(x / a_{n}\right)^{2}}} d x=a_{n} \pi f_{n}(t)
$$

In order to verify this method, we will calculate the distribution functions of currents over the vibrators of a three-element AR based on chiral metamaterials and compare them with the calculation results obtained in the FEKO software package.

\section{Verification of MEAA analysis method}

This MEAA represents three half-wave symmetric vibrators, the length of which is $l_{1} / \lambda=l_{2} / \lambda=l_{3} / \lambda=0.25$ and the width is $a_{1} / \lambda=a_{2} / \lambda=a_{3} / \lambda=0.025$. The vibrators are located at a distance of $\Delta_{1} / \lambda=\Delta_{2} / \lambda=1$ from each other on a dielectric substrate, height of $d / \lambda=0.1$ and metallized on one side. The permittivity and permeabilities of the substrate are equals to $\varepsilon_{1}=\mu_{1}=1$.

Fig. 2 shows the results of calculating the current distribution functions for each emitter in common-mode excitation.

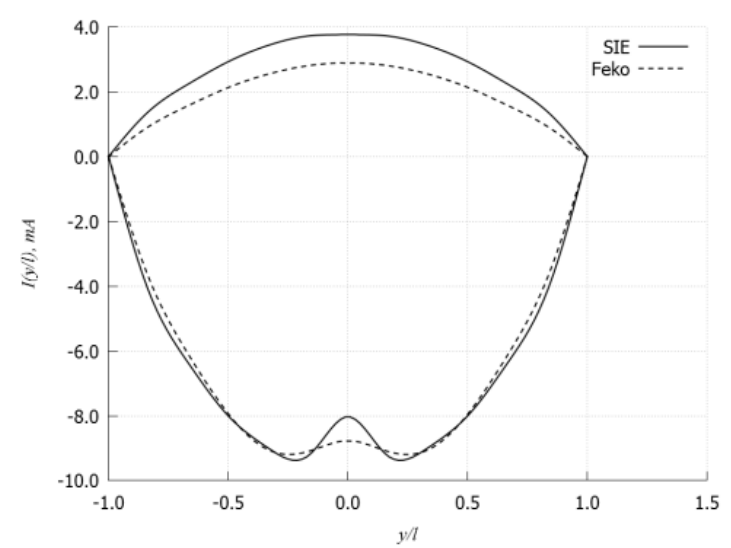

(a)

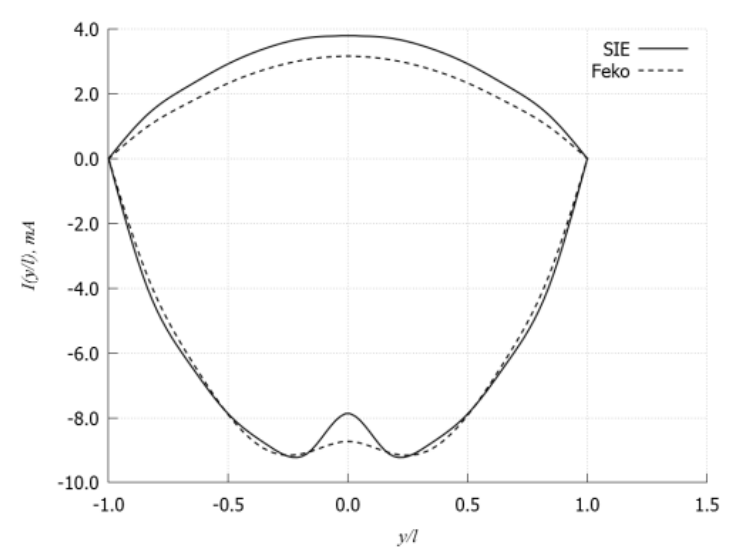

(b)

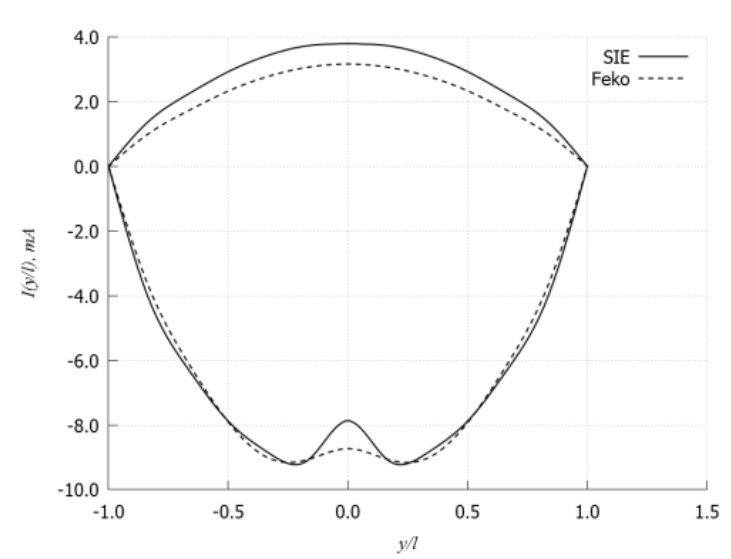

(c)

Fig. 2. Current distribution functions for the first (a), second (b) and third (c) vibrators with in-phase excitation

\section{Numerical results and discussion}

Fig. 3 shows the dependences of the input impedance on a three-element strip AR with a chiral metamaterial 
substrate made on the basis of left and right spirals. AR parameters are similar to the previous case. The chirality parameter is $\chi=0.3$. The dependencies are given for the excitation of only one vibrator.

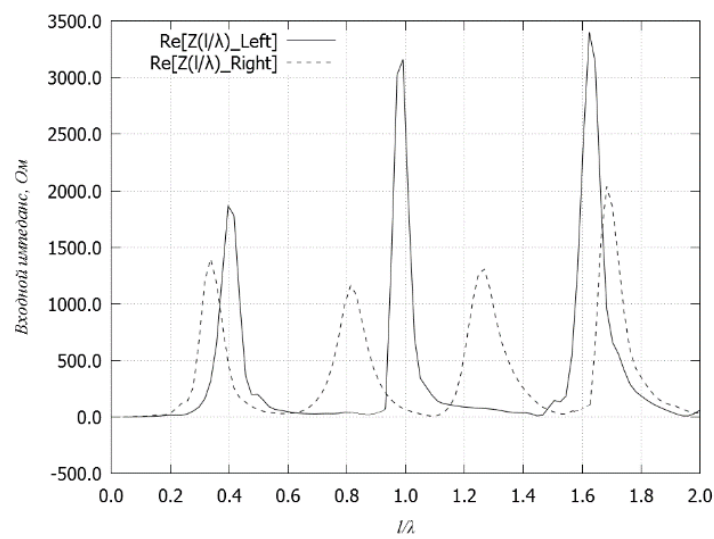

(a)

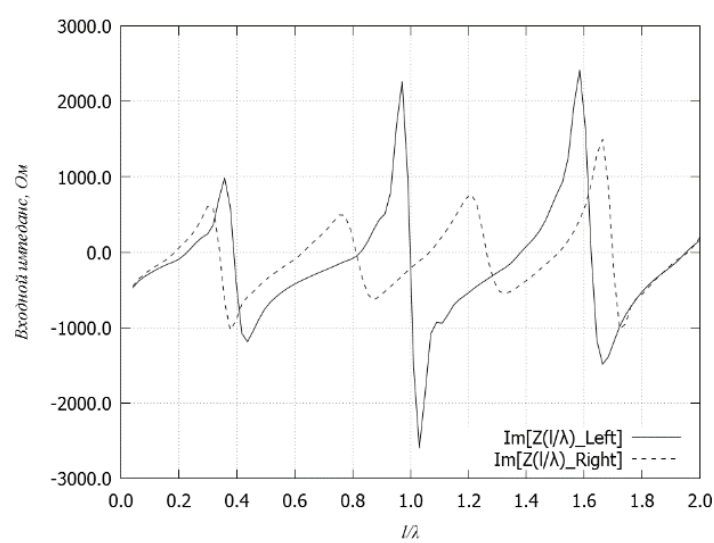

(b)

Fig. 3. Dependences of the real (a) and imaginary (b) parts of the input impedance of the first emitter from $l / \lambda$ upon excitation of one vibrator

We have also calculated the effect of a substrate of chiral metamaterial on the isolation between the vibrators of three-element MEAA. Fig.4 4 shows the dependence of the isolation between the emitters of a three-element AR on the chirality parameter for the cases when (a) only one vibrator is excited and two vibrators (b) are excited.Here , index 1-2 means that the EMF source is installed on the vibrator 1 , and the load on the vibrator 2. Similarly, index 1-3 means the presence of EMF sources on vibrators 1 and 3 , and the load on vibrator 2. Parameters of the $\mathrm{AR}$ are equal to: $\Delta_{1} / \lambda=\Delta_{2} / \lambda=0.25$ and $l / \lambda=0.5$. It is important to note that zeros value of the chirality parameter corresponds to a substrate made of a dielectric, negative values correspond to $\chi$ substrate based on left-handed chiral elements, and positive values correspond to righthanded chiral elements.

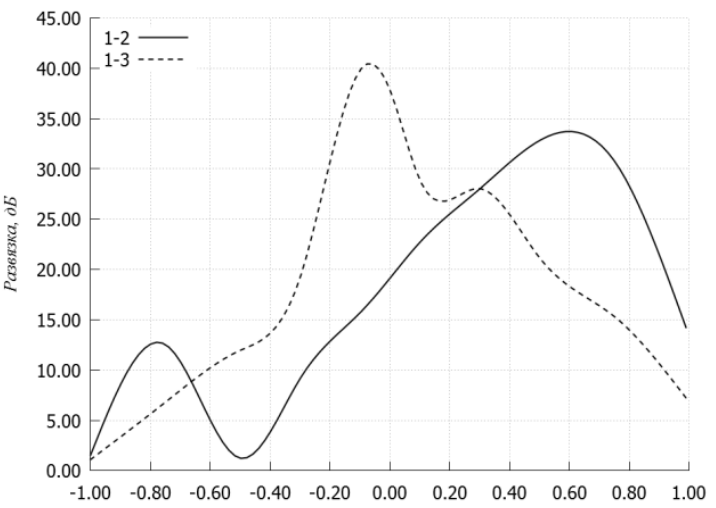

(a)

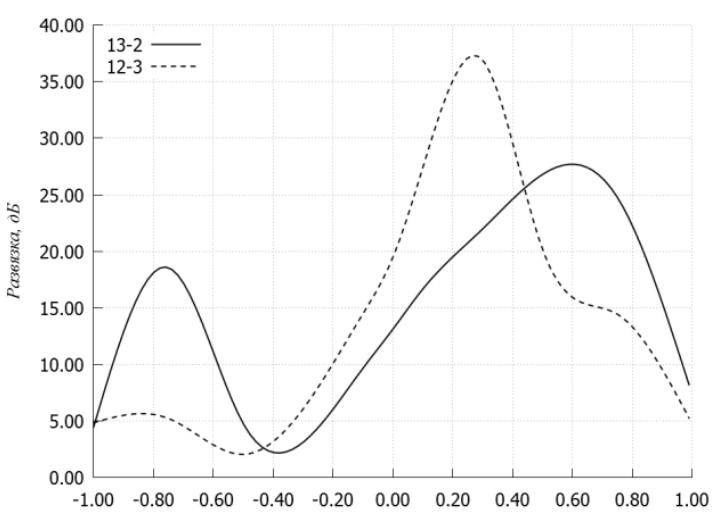

(b)

Fig. 4. Dependence of the isolation between the emitters of a three-element MEAA on the chirality parameter

As it can be seen from their graphs, the decoupling values for MEAA with a substrate of chiral metamaterials at certain values of the chirality parameter are significantly higher than those for MEAA with dielectric.

To conclude, his study presents the method of electrodynamic analysis of multi-element strip MEAA based on chiral metamaterials. A system of singular integral equations with respect to unknown distribution functions of current density over emitters has been obtained. The method was tested by comparing the obtained current distributions with the results calculated in FEKO. It has been shown that the use of chiral substrates in MEAA can significantly improve the isolation between the emitters.

Acknowledgment. The authors prepared this article under the support of the project "Leading Talents of Guangdong Province", № 00201502 (2016-2020) in the Jinan University (China, Guangzhou).

\section{References}

1. N. Engheta, Proc. of the 1988 URSI, 213 (1988)

2. A. Erentok, R.W. Ziolkowski, IEEE Trans. on Ant. and Propag. 3, 691-707 (2008)

3. V.V. Badalov, S.O. Belyaev, D.A. Kopylov, A.M. Nescheret, Ant. 11, 31-38 (2017) [in Russian] 
4. A.N. Bespalov, A.L. Buzov, D.V. Mishin, D.S. Klyuev, A.M. Nescheret, Radio Eng. 3, 5-11 (2019) [in Russian]

5. M. Alibakhshikenari, M. Khalily, B.S. Virdee, C.H. See, R. Abd-Alhameed, E. Limiti, IEEE Access, DOI 10.1109/ACCESS.2019.2899326.

6. A.A. Potapov, V.I. Grachev, Proc. PIERS 2012 in Moscow. 1584 - 1587 (2012).

7. A.A. Potapov Proc. 7th Int. Conf. "Neural Networks and Artificial Intelligence”. 74 - 77 (2012)

8. J. Xiang, S. He, Y. Zhang and G. Zhu, Int. J. of Appl. Electromag. and Mech. 48(1), 33-46 (2015)

9. C. Zebiri, M. Lashab and F. Benabdelaziz, IET MW, Ant. Propag. 5(1), 17-29 (2011)

10. C. Zebiri, S. Daoudi, F.Benabdelaziz, M. Lashab, D. Sayad, N.T. Ali and R.A. Abd-Alhameed, Int. J. of Appl. Electromag. and Mech. 51, 249-260 (2016)

11. M. A. Buzova, A.N. Dementyev, D.S. Klyuev, et al, Doklady Physics. 6, 231-234 (2018)

12. A.L. Buzov, D.S. Klyuev, D.A. Kopylov, A.M. Nescheret, Tech. Phys. Letters, 12, 1065-1068 (2018)

13. A.L. Buzov, M.A. Buzova, D.S. Klyuev, D.V. Mishin, A.M. Neshcheret, J. of Comm. Tech. and Elect. 11, 1259-1264 (2018)

14. I.V. Lindell, IEEE Trans Microwave Theory Tech. 40(2), 402-405 (1992)

15. V. Lindell, A.H. Sihvola, S.A. Tretyskov and A.J. Vitanen, Media Norwood, MA: Altech House. (1994) 